\title{
Long non-coding RNA HOTAIR: A novel oncogene (Review)
}

\author{
XIN YU ${ }^{1,2 *}$ and ZHENG LI ${ }^{1 *}$ \\ ${ }^{1}$ Department of Orthopedic Surgery, Peking Union Medical College Hospital, Peking Union Medical College, Beijing 100730; \\ ${ }^{2}$ State Key Laboratory of Cardiovascular Disease, Fuwai Hospital, National Center for Cardiovascular Diseases, \\ Chinese Academy of Medical Sciences and Peking Union Medical College, Beijing 100037, P.R. China
}

Received July 29, 2014; Accepted April 20, 2015

DOI: $10.3892 / \mathrm{mmr} .2015 .4161$

\begin{abstract}
Long non-coding RNAs (IncRNAs) have been found to be pervasively transcribed in the genome and are critical regulators of the epigenome. Increasing evidence suggests that IncRNAs are aberrantly expressed in several types of human cancer and that they are important in the initiation, development and metastasis of human cancer. Previous studies have revealed that HOX transcript antisense intergenic RNA (HOTAIR) was frequently upregulated in various types of cancer, including breast cancer, esophageal cancer, lung cancer and gastric cancer. In addition, patients with high expression levels of HOTAIR have a significantly poorer prognosis, compared with those with low levels of expression. HOTAIR is involved in the control of cell apoptosis, growth, metastasis, angiogenesis, DNA repair and tumor cells metabolism. The present review provides an overview of the current knowledge concerning the role of HOTAIR in tumor development and progression.
\end{abstract}

\section{Contents}

1. Introduction

2. Structure and biological function of HOTAIR

3. HOTAIR status in human malignancies

4. Mechanisms of HOTAIR in tumorigeneses

5. Regulation of HOTAIR expression

6. Implications in cancer management

7. Conclusions and future directions

Correspondence to: Dr Zheng Li, Department of Orthopedic Surgery, Peking Union Medical College Hospital, Peking Union Medical College, 1 Shua Fu Yuan, Beijing 100730, P.R. China E-mail:kleeo@163.com

${ }^{*}$ Contributed equally

Key words: HOTAIR, long non-coding RNAs, oncogene, cancer

\section{Introduction}

An oncogene is defined as a mutated gene, whose product contributes to the initiation or progression of cancer (1). Activation of oncogenes are critical in the molecular pathogenesis of human neoplasms (2). Known oncogene, including Ras, BRAF, $\beta$-catenin, and Myc, are predominantly protein-coding genes, the functions of which are mediated by their gene products-proteins (3-6). Genome sequencing projects have revealed that the human genome contains $<2 \%$ protein coding genes, while $>90 \%$ of the genome is transcripted into non-coding (nc)RNAs $(7,8)$. Based on nucleotide size, ncRNAs can be classified into two major classes: Small ncRNAs and long non-coding RNAs (lincRNAs) (9-11). Small ncRNAs include the well-documented microRNAs. Several microRNAs are increased in multiple types of cancer which exhibit pro-oncogenic activity through the induction of oncogenes or the inhibition of multiple genes with tumor suppressor-like activity (12-14). LincRNAs are mRNA-like transcripts consisting of $>200$ nucleotides. Increasing evidence has reported the deregulation of lincRNAs across numerous types of cancer, indicating that lincRNA may be involved in tumorigenesis (15). Several lincRNAs act as scaffolds, which regulate molecular (protein, RNA and DNA) interactions that are required for various signaling networks, and is accomplished partly through association with chromatin-modifying complexes (15-17).

Hox transcript antisense intergenic RNA (HOTAIR) was one of the first lincRNAs reported to be involved in the development of cancer (18). HOTAIR is transcribed from the HOXC locus but represses expression in the more distal HOXD locus and genes on other chromosomes, leading to the decreased expression of multiple genes, particularly the metastasis suppressing genes (18-20). HOTAIR is the first lincRNA identified to regulate genes at a distance (18). HOTAIR is overexpressed in several types of malignancy, including breast cancer, gastric cancer, liver cancer and sarcoma (21-24). Furthermore, in vitro analysis has demonstrated that HOTAIR can promote cancer cells proliferation, invasion and metastasis, while inhibit apoptosis (25). Notably, in murine xenograft models, HOTAIR-knockout can reduce tumor growth in vivo $(26,27)$. As a result, HOTAIR has been suggested as a potential oncogene.

The present review aims to discuss the current knowledge of the properties of HOTAIR, its status in human tumors, the 
mechanism in tumorigenesis and their potential implications in cancer management.

\section{Structure and biological function of HOTAIR}

HOTAIR, first identified from a custom-tilling array of the HOXC locus, is encoded from the HOXC locus on chromosome $12 \mathrm{q} 13.13(18,28)$. It belongs to the long non-coding RNAs, with 2,158 nucleotides. HOTAIR RNA does not encode any proteins, however it is important in gene regulation by modifying chromatin structure $(16,18,20)$.

Polycomb proteins are a group of proteins involved in the repression of transcription of thousands of genes, which are critical in differentiation, maintenance of cell identity and cancer development $(20,29,30)$. Polycomb proteins act in several protein complexes, polycomb repressive complex (PRC) 1 and PRC2. PRC2 is a histone H3 lysine 27 (H3K27) methylase and, by modulating $\mathrm{H} 3 \mathrm{~K} 27$ methylation, PRC2 is involved in developmental gene silencing and cancer progression $(28,31)$. The association between lincRNA and plycomb proteins to induce silencing is considered a common mechanism in the epigenetic regulation of 1 ncRNAs, including HOTAIR. The core PRC2 cannot target and silence genomic regions alone. Instead, HOTAR binding is required to guide PRC2 to the specific regions of the genome, where PRC2 associates and epigenetically silences the gene expression. HOTAR binding results in genome-wide retargeting of PRC2. HOTAIR contains two independently binding domains, 5' and $3^{\prime}$ domains, which bind the PRC2 and lysine-specific demethylase 1 (LSD1) complexes, respectively. The HOTAIR-PRC2-LSD1 complex then targets the HOXD locus on chromosome 2, silencing the genes involved in the suppression of metastasis (32), as shown in Fig. 1. It is clear that HOTAIR reprograms the chromatin to promote cancer metastasis. However, the precise mechanism of the activities of HOTAIR remains to be elucidated.

\section{HOTAIR status in human malignancies}

Since its identification in breast cancer, the aberrant expression of HOTAIR has been reported in various types of human cancer (22-24). IncRNA HOTAIR is an independent prognostic marker of metastasis in estrogen receptor-positive primary breast cancer. HOTAIR is overexpressed in multiple types of cancers, and its overexpression is associated with metastasis and poor survival rates. The regulation and function of HOTAIR in cancer is summarized in Table I.

HOTAIR and breast cancer. A significantly higher level of HOTAIR expression is observed in primary and metastatic breast cancer, as compared with normal breast epithelium, and HOTAIR is an independent biomarker for predicting the risk of metastasis and mortality in breast cancer (20). Overexpression of HOTAIR increases the invasive ability of breast cancer cells in vitro and in vivo (20). High expression levels of HOTAIR correlate positively with DNA methylation in primary breast cancer (33). Methylation has been associated with poor disease prognosis, however, no significant associations have been identified between the expression of HOTAIR and clinical outcomes, indicating that HOTAIR may not be an independent prognostic biomarker in breast cancer (33).
Sorensen et al demonstrated that high expression levels of HOTAIR correlated with decreased prognosis, serving as an independent predictor of metastasis in patients with estrogen receptor (ER)-positive breast cancer, but not those with ER-negative breast cancer (34).

HOTAIR and esophageal cancer. HOTAIR is upregulated in esophageal squamous cell cancer (ESCC). A positive correlation exists between high levels of HOTAIR and clinical stage, serving as an independent prognostic factor in ESCC patients and cell lines $(35,36)$. Higher expression levels of HOTAIR are associated with advanced disease stage, a poorer prognosis and reduced survival rate (36). Depletion of HOTAIR in ESCC cells reduces proliferation, foci formation, migration and invasion of the extracellular matrix, alters cell cycle progression and increases the sensitivity of cells to apoptosis in vitro $(35,37)$. In addition, microarray analysis has revealed that HOTAIR reprograms the gene expression profile in ESCC cells, leading to an increase in genes involved in tumorigenesis, including genes regulating migration and cell cycle (37). HOTAIR can also decrease the expression of Wnt inhibitory factor 1 (WIF-1), which is important in cell proliferation, migration and tumor progression (36). The HOTAIR/WIF-1 axis provides a potential molecular mechanism of HOTAIR in the pathogenesis of ESCC (36).

HOTAIR and lung cancer. Using reverse transcription-quantitative polymerase chain reaction (RT-qPCR) analysis in 42 non-small cell lung cancer (NSCLC) samples, HOTAIR levels were found to be significantly higher in the cancerous tissues, compared with normal lung cells, correlating positively with the pathological stage and lymph node metastasis $(38,39)$. HOTAIR is a prognostic parameter for survival rates, and higher expression levels of HOTAIR are associated with a poorer prognosis in patients with NSCLC $(39,40)$. In addition, subsequently functional analysis in vitro has revealed its mediation in the migration and invasion of NSCLC cell lines. HOTAIR knockdown was observed to affect the level of HOXA5, which inhibited the migration and invasion of NSCLC cells, indicating that HOTAIR may partially exert its effects through the regulation of HOXA5 $(38,39)$. HOTAIR is induced by type I collagen (Col-1), which was a type of interstitial extracellular matrix (ECM), aberrantly enriched in the tumor microenvironment (39).

HOTAIR and gastric cancer. The expression level of HOTAIR are significantly increased in gastric cancer tissues, significantly correlating with lymph node metastasis and tumor-node-metastasis stage $(22,41)$. High expression levels of HOTAIR predict poorer overall survival rates in patients with gastric cancer (41). In addition, gastric cancer cells expressing HOTAIR exhibit higher proliferation ability in vitro and a higher rate of liver metastasis (22). Loss of functional analysis has revealed the importance of HOTAIR in gastric cancer cell invasion in vitro. The expression of MMP1 and MMP3, which can favor metastasis by remodeling the ECM and degrading the basement membrane, are also inhibited by HOTAIR knockdown (41).

HOTAIR and liver cancer. HOTAIR is significantly upregulated in hepatocellular carcinoma (HCC) tissues compared with 
Hox Genes

Hox Genes HOXC Locus

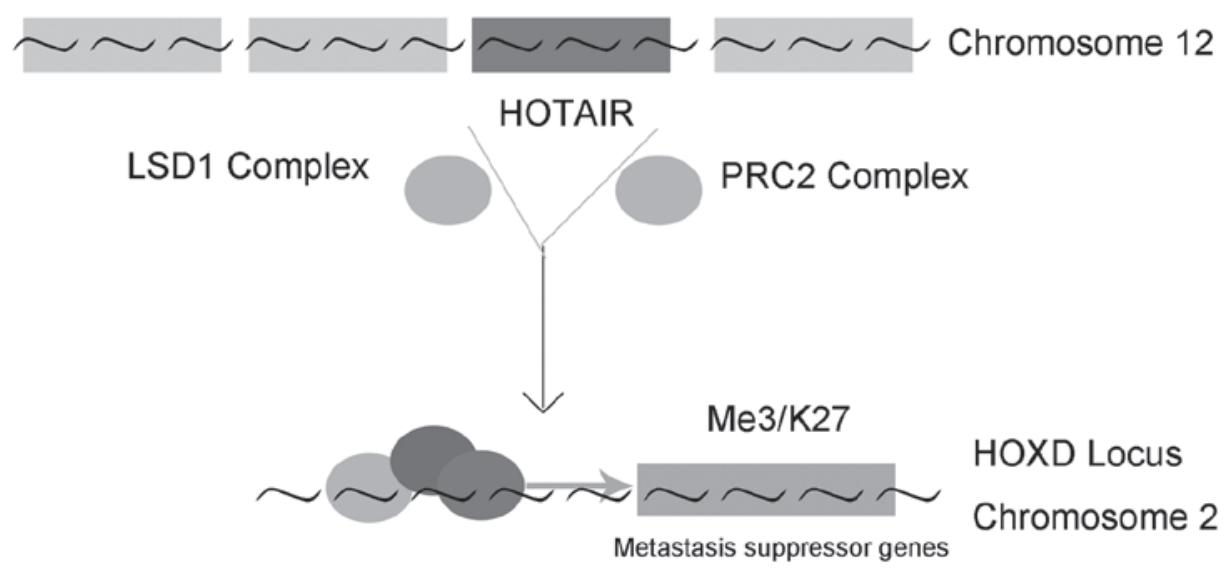

Figure 1. Proposed mechanism of HOTAIR-mediated gene silencing of $40 \mathrm{~kb}$ of the HOXD locus, which is involved in developmental pattering. The HOTAIR lncRNA is transcribed from the HOXC locus and functions in the binding, and the recruitment and binding of the LSD1 and PRC2 complex to the HOXD locus. Through an undetermined mechanism, the HOTAIR-PRC2-LSD1 complex is redirected to the HOXD locus on chromosome 2, where genes involved in metastasis suppression are silenced through H3K27 methylation and H3K4 demethylation. HOTAIR, HOX transcript antisense intergenic RNA; lncRNA, long non-coding RNA; PRC. polycomb repressive complex; LSD1, lysine-specific demethylase 1.

adjacent normal tissues (23). The expression of HOTAIR is an independent prognostic factor for HCC recurrence following liver transplantation (23). Patients with higher expression levels of HOTAIR have been observed to have increased size and reduced prognosis, compared with those with lower expression levels of HOTAIR (42). Depletion of HOTAIR in HepG2 cells decreases cell viability and invasiveness, and increases the sensitivity to tumor necrosis factor- $\alpha$-induced apoptosis and chemotherapy (23). In addition, the introduction of HOTAIR into liver cancer cells increased the proliferation rate in vitro (42). These results indicate the functional role of HOTAIR in the progression of liver cancer.

HOTAIR and endometrial cancer. The levels of HOTAIR are significantly higher in endometrial cancer (EC) tissues than the normal tissues (43). A positive correlation has been observed between the expression of HOTAIR and the EC grade, depth of the myometrium, lymphovascular invasion and lymph node metastasis (43). Similar to the types of cancer mentioned above, the expression of HOTAIR is also a predictor of poor prognosis in patients with EC (43).

HOTAIR and prostate cancer. The expression of HOTAIR is upregulated in castration-resistant prostate cancer ( $\mathrm{PCa}$ ) cell lines, compared with normal prostate cells. Knockdown of the expression of HOTAIR by small interfering RNA leads to a reduction in PCa cell proliferation, migration and invasion, and increased apoptosis and cell cycle arrest (44).

HOTAIR and nasopharyngeal cancer. The expression of HOTAIR is higher in nasopharyngeal cancer, compared with non-cancerous tissue, which is positively associated with tumor size, clinical stage and lymph node burden. Furthermore, HOTAIR overexpression has been associated with significantly decreased survival rates (45). In addition, in vitro analysis has demonstrated that HOTAIR is important in the proliferation, migration and invasion of nasopharyngeal cancer cells (45).
HOTAIR and laryngeal cancer. According to qPCR analysis, HOTAIR is significantly overexpressed in laryngeal squamous cell carcinoma, compared with adjacent non-neoplastic tissue (26), and its expression level is higher in high-grade carcinoma than in low-grade carcinoma. Furthermore, patients with higher expression levels of HOTAIR have been observed to have a relatively poorer prognosis. In vitro analysis demonstrated that knockdown of HOTAIR in the Hep-2 cells inhibited the invasion and promoted apoptosis (26). In addition, HOTAIR knockout murine xenograft models reduce the growth of laryngeal squamous cell carcinoma tumors in vivo. Knockdown of HOTAIR in Hep-2 cells resulted in a significant decrease in the methylation level of PTEN, which is a tumor suppressor gene involved in various types of human malignancy. Therefore, the epigenetic modification of PTEN may be the underlying molecular mechanism for tumorigenesis by HOTAIR (26).

HOTAIR and pancreatic cancer. The expression of HOTAIR is high in pancreatic cancerous tissue, compared with non-cancerous tissue $(27,46)$. Its expression level is also higher in high-grade carcinoma than in low-grade carcinoma. In addition, the expression of HOTAIR is significantly increased in primary pancreatic tumors, which have metastasized to lymph nodes, compared with those that are localized in the pancreas only (27). Consistent with the findings in other cancer cell lines, HOTAIR depletion in Panc1 and L3.6pL pancreatic cancer cells inhibits cell proliferation, alters cell cycle progression and induces apoptosis, indicating its functional role in the progression of pancreatic cancer (27). Of note, in murine xenograft models, HOTAIR knockout in the pancreatic cancer cells inhibited tumor growth in vivo, further demonstrating the pro-oncogenic function of HOTAIR in pancreatic cancer (27). HOTAIR knockdown resulted in changes in the expression of 1,006 genes, which may contribute to the functional pro-oncogenic activity of HOTAIR in Pancl cells (27).

HOTAIR and colorectal cancer. qPCR analysis has revealed that the expression levels of HOTAIR are higher in colorectal 
Table I. Changes in the expression of HOTAIR in different types of human cancer.

\begin{tabular}{lccc}
\hline Type of cancer & Expression & Effect on invasion/metastasis & Reference \\
\hline Breast cancer & Increased & Promote & 20,33 \\
Esophageal cancer & Increased & Promote & $35,36,37$ \\
Lung cancer & Increased & Promote & $38,39,40$ \\
Gastric cancer & Increased & Promote & 22,41 \\
Liver cancer & Increased & Promote & 23,42 \\
Endometrial cancer & Increased & Unknown & 43 \\
Prostate cancer & Increased & Promote & 44 \\
Nasopharyngeal cancer & Increased & Promote & 45 \\
Laryngeal cancer & Increased & Promote & 26, \\
Pancreatic cancer & Increased & Promote & 27,46 \\
Colorectal cancer & Increased & Unknown & 47 \\
Melanoma & Increased & Promote & 48 \\
Glioma & Increased & Promote & 49 \\
Sarcoma & Increased & Promote & 24 \\
Pituitary adenoma & Increased & Unknown & 50 \\
\hline
\end{tabular}

cancer compared with non-tumor tissues (47). Furthermore, HOTAIR has also been revealed as a negative prognostic factor for patients with colorectal cancer (47). The expression levels of HOTAIR were found to be higher in tumors, which metastasized to the liver, compared with those that did not metastasize; indicating that the upregulation of HOTAIR may be a critical element in the metastatic progression of colorectal cancer (47).

HOTAIR and melanoma. Melanoma is a type of skin cancer with high potential to metastasize to the vital organs. HOTAIR has been demonstrated to be involved in the metastasis of melanoma. Expression levels of HOTAIR are higher in metastatic lymph nodes than in corresponding primary melanoma (48). Furthermore, in vitro analysis has revealed that HOTAIR depletion decreases the motility and invasion of human melanoma cells and inhibits degradation of the gelatin matrix (48).

HOTAIR and glioma. Glioma is the most common primary tumor in the brain (49). Expression levels of HOTAIR are higher in high-grade glioma, compared with low-grade glioma. Higher levels of HOTAIR were identified as an indicator for reduced survival rates, serving as an independent overall survival factor (49). Gene set enrichment analysis has revealed that the expression of HOTAIR modulates gene sets involved in cell cycle progression, which may be the mechanism underlying the pro-oncogenic activity of HOTAIR (49).

HOTAIR and sarcoma. Sarcomas consist of a heterogenous group of soft tissue and bone malignant tumors (24). Expression of HOTAIR has been detected in the primary and metastatic sarcoma tissues using western blotting and RT-qPCR analysis. In addition, high expression levels of HOTAIR have been correlated with a high probability of metastasis in primary sarcoma. Of note, the inhibition of HOTAIR has been observed to result in a good response to treatment, in terms of necrosis (24).
HOTAIR and pituitary adenomas. Pituitary adenomas belong to primary intracranial tumors, the majority of which secrete pituitary hormones that result in increased levels of blood hormones and clinical syndromes. A non-functioning pituitary adenoma (NFPA) is defined as a pituitary adenomas, which does not cause clinical hormone hypersecretion. The majority of pituitary adenomas are histologically benign; however, they have a marked ability to invade surrounding structures. The expression of HOTAIR has been reported to increase between normal anterior pituitaries, non-invasive NFPAs and invasive NFPAs, indicating a potential role of HOTAIR in NFPA invasion (50).

In conclusion, a substantial number of studies have revealed that HOTAIR is upregulated in multiple types of human cancer, and its overexpression is correlated with metastasis and poor prognosis. HOTAIR may serve as a potential tumor marker and therapeutic target in the future. Further investigations are required to clarify its expression in other types of cancer, particularly of the molecular mechanisms associated with the suppression and activation of genes by HOTAIR in cancer.

\section{Mechanisms of HOTAIR in tumorigenesis}

Epithelial-to-mesenchymal transition (EMT) and maintenance of cancer stem cells (CSCs). The EMT is a multistep process by which epithelial cells lose epithelial characteristics and gain mesenchymal characteristics, including motility and invasive properties (51). The metastatic process involves several steps. EMT is considered to be the first step of metastatic spread. Dissociation of cells from the epithelial layer results in the deregulation of intercellular contacts and the acquisition of migratory abilities.

CSCs are pluripotent tumor-initiating cells, which have the ability to self-renew. Due to their unique characteristics, CSCs are considered the basis for tumor initiation, development, metastasis and recurrence (52). 


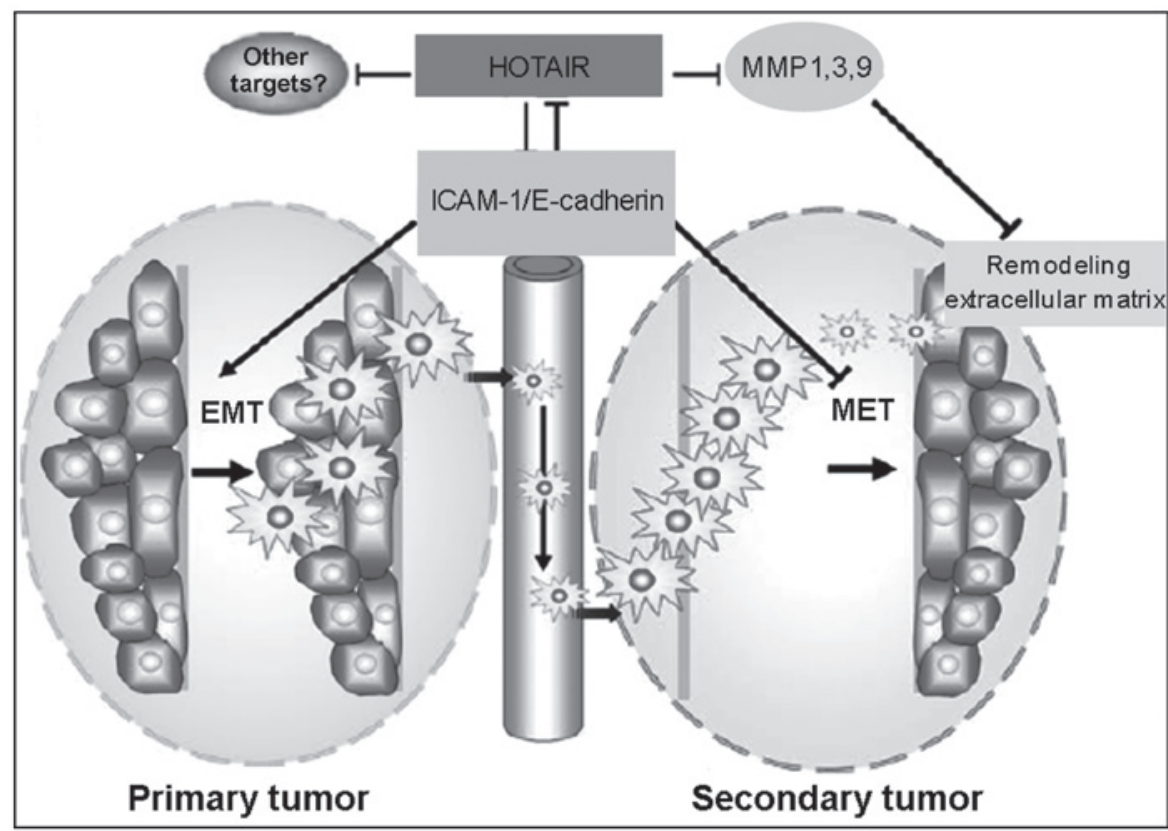

Figure 2. HOTAIR regulates EMT/MET and cancer metastasis. HOTAIR targets ICAM-1, E-cadherin, MMP1, MMP3 and MMP9, and is important at the early and late stages of metastasis. HOTAIR, HOX transcript antisense intergenic RNA; EMT, epithelial-mesenchymal transition; MET, mesenchymal-epithelial transition; ICAM, intercellular adhesion molecule 1; MMP, matrix metalloproteinase.

The overexpression of HOTAIR has been reported to induce EMT and support CSCs, with HOTAIR silencing leading to the failure of EMT and CSC establishment (53). In addition, in vitro analysis has demonstrated that suppression of HOTAIR can reverse EMT process in gastric cancer cells (41). The molecular mechanism by which HOTAIR induces EMT remains to be elucidated. The overexpresion of HOTAIR increases the expression of snail, which is a potent EMT inducer (53). HOTAIR can also accelerate the degradation of Ataxin-1, which can activate the promoter of E-cadherin. E-cadherin is the key regulator of the EMT, as it regulates epithelial cell-to-cell interactions (54).

It has been suggested that HOTAIR may be involved in EMT/CSC establishment by regulating certain critical molecular signaling pathways. A potential explanation may be found in the HOTAIR targeting of intercellular adhesion molecule (ICAM)-1 and members of the matrix metalloproteinase (MMP) family, including MMP1, MMP3 and MMP9 in gastric cancer (41). MMP1 and MMP3 have been reported to be suppressed by HOTAIR knockdown (41), and MMPs can favor metastasis by remodeling the ECM and degrading the basement membrane. In addition, HOTAIR facilitates the ubiquitination and, thus, accelerates the degradation of Ataxin-1. Ataxin-1 has been demonstrated to activate the promoter of E-cadherin, which is a key tumor suppressor that suppresses the invasiveness of cancer cells (55-57). These findings indicate that HOATIR may promote metastasis through the regulation of the metastasis-associated genes, including MMPs and Ataxin-1 (Fig. 2).

HOTAIR and the control of gene transcription. Several genes are induced or repressed by HOTAIR, including WIF-1, HOXD10, MMP1/3, PTEN and snail (20,36,41). In esophageal cancer, HOTAIR decreases the expression of WIF-1 (36), which is critical in cell proliferation, migration and tumor progression. HOTAIR directly decreases the expression of WIF-1 by promoting its histone, H3K27, methylation in the promoter region, activating Wnt/b-catenin signaling pathway. The Wnt/b-catenin signaling pathway is important in mediating cell proliferation and migration, and in controlling tumor progression, and ha been found to be aberrantly activated in multiple types of cancer, including $\operatorname{ESCC}(5,58)$. In breast cancer, the overexpression of HOTAIR leads to epigenetic silencing of multiple genes, including HOXD10 and other metastasis suppressor genes (20). In gastric cancer, as mentioned above, overexpression of HOTAIR is associated with the increase of ICAM-1 and certain members of the MMPs family including MMP1, MMP3, and MMP9 (41), indicating that HOATIR may promote metastasis through the regulation of MMPs.

A significant role of lncRNAs is in the regulation of gene expression via a mechanism involving interaction with the epigenetic silencing complex, polycomb repressive complex 2 (PRC2), with $20 \%$ of all lncRNA transcripts estimated to bind PRC2 $(20,47)$. Modulation of HOTAIR alters the levels of various genes in several cancer cells, the majority of which are PRC2-dependent $(20,47)$. HOTAIR-induced PRC2 target genes include JAM2, PCDH10 and PCDHB5, and the majority are positive regulators of cancer metastasis (20). In addition, the modulation of the expression of these genes and the metastatic effect of HOTAIR can be reversed by simultaneous PRC2 depletion, revealing a HOTAIR-polycomb pathway in cancer invasion (46). HOTAR binding is required to target PRC2 to the specific regions of the genome. As a H3K27 methylase, PRC2 can modulate H3K27 methylation. Methylation of H3K27 leads to transcriptional repression and is, therefore, involved in controlling gene expression patterns (59). Deregulation of H3K27 methylation patterns are also commonly observed in multiple types of cancer (60). 
Ubiquitination is a process commonly leading to the proteasome-mediated degradation of the target protein (61). Ubiquitination is one of the most important post-translational modifications, with a pivotal role in tumor development, and can regulate tumor suppressors and oncogenes. HOTAIR can control protein levels by promoting ubiquitin-mediated proteolysis (62). HOTAIR facilitates the ubiquitination of Ataxin-1 and Snurportin-1, in cells and in vitro, by associating with E3 ubiquitin ligases bearing RNA-binding domains, Dzip3 and Mex3b, as well as with their respective ubiquitination substrates, Ataxin-1 and Snurportin-1. In this manner, HOTAIR can accelerate the degradation of Ataxin-1 and Snurportin-1. Ataxin-1 has been demonstrated to activate the promoter of E-cadherin, a key tumor suppressor that suppresses the invasiveness of cancer cells (55-57).

In conclusion, by binding PRC2, HOTAIR can regulate the expression of genes that are conducive to cell motility, matrix invasion and remodeling the chromatin state in cancer cells.

\section{Regulation of HOTAIR expression}

As mentioned above, HOTAIR is frequently increased in a variety of human malignances. However, the mechanism leading to its deregulation in human cancer remains to be elucidated. In lung cancer cells, HOTAIR is induced by type I collagen (Col-1), a type of interstitial ECM that is aberrantly enriched in the tumor microenvironment, indicating that the expression of HOTAIR in cancer cells may result from the response of the cancer cells to Col-1 (63). In breast cancer, bisphenol-A (BPA) and diethylstilbestrol (DES) induce HOTAIR expression in cultured human breast cancer cells and in vivo in the mammary glands of rats (64). BPA and DES exposure induce HOTAIR expression by altering the epigenetic programming of the HOTAIR promoters. In the presence of BPA and DES, HOTAIR promoter estrogen-response-elements (EREs) are bound by ERs and ER co-regulators, their chromatin is modified via histone methylation and acetylation, and they are activated by HOTAIR.

\section{Implications in cancer management}

As the overexpression of HOTAIR has been documented in a number of human malignancies, it provides a promising approach for anti-cancer therapies. Firstly, HOTAIR can be used as a biomarker for cancer diagnosis. High expression levels of HOTAIR are correlated with enhanced metastasis and is presents a negative prognostic factor for patient survival rates. By monitoring the levels of HOTAIR in certain types of tumor, including alterations in gene or protein levels, the risk of tumor development and progression, and the prognosis of the tumor, can be predicted. The observation in murine xenograft models that HOTAIR knockout can reduce tumor growth in vivo $(26,27)$, knockout of the HOTAIR gene or decreasing the protein level of HOTAIR may provide a promising target for cancer therapy.

\section{Conclusions and future directions}

Since its first observation, high expression levels of HOTAIR in various types of human tumor have been well documented.
High expression levels of HOTAIR correlate with enhanced metastasis in cancer, and overexpression of HOTAIR is associated with poorer prognosis in cancer patients, most notably due to the increased metastasis.

To further clarify its role, the expression of HOTAIR in other types of cancer requires investigation. Although the expression of HOTAIR has been extensively investigated in various types of cancer, the molecular mechanism underlying the effects of HOTAIR in tumorigenesis remain to be elucidated. It appears that HOTAIR is involved in modulating the cancer epigenome and reprogramming the chromatin state, which is accomplished through the interaction with PRC2. HOTAIR binding can induce PRC2 to specific regions of the genome, which can repress or promote the transcription of genes, including WIF-1, HOXD10, MMP1/3, PTEN and snail. The majority of these genes regulate cancer cell proliferation and invasion, and are associated with tumor progression. By binding PRC2, HOTAIR can regulate gene expression and remodel chromatin states, which is conducive to cell motility and matrix invasion in cancer cells.

The molecular mechanism underlying the upregulation of HOTAIR in cancer also requires further investigation, to determine which transcription factors or signaling pathways mediate HOTAIR induction in cancer, In lung cancer cells, HOTAIR is induced by Col-1, which is a type of ECM aberrantly enriched in the tumor microenvironment. Col-1 has been demonstrated to promote the development of cancer, indicating that the expression of HOTAIR in cancer cells may result from the response of cancer cells to Col-1 (63). However, further investigations are required to fully elucidated the function of this highly expressed molecule in cancer.

\section{Acknowledgements}

The present study was supported by the National Natural Science Foundation of China (grant no. 81401847).

\section{References}

1. Kim H, Huang W, Jiang X, Pennicooke B, Park PJ and Johnson MD: Integrative genome analysis reveals an oncomir/oncogene cluster regulating glioblastoma survivorship. Proc Natl Acad Sci USA 107: 2183-2188, 2010.

2. Zhang B, Pan X, Cobb GP and Anderson TA: microRNAs as oncogenes and tumor suppressors. Dev Biol 302: 1-12, 2007.

3. Young A, Lou D and McCormick F: Oncogenic and wild-type Ras play divergent roles in the regulation of mitogen-activated protein kinase signaling. Cancer Discov 3: 112-123, 2013.

4. Thiel A and Ristimaki A: Toward a molecular classification of colorectal cancer: the role of BRAF. Front Oncol 3: 281, 2013.

5. MacDonald BT, Tamai $\mathrm{K}$ and $\mathrm{He} \mathrm{X}$ : Wnt/beta-catenin signaling: components, mechanisms and diseases. Dev Cell 17: 9-26, 2009.

6. Shimizu T, Ishikawa T, Sugihara E, et al: c-MYC overexpression with loss of Ink4a/Arf transforms bone marrow stromal cells into osteosarcoma accompanied by loss of adipogenesis. Oncogene 29: 5687-5699, 2010.

7. Stein LD: Human genome: end of the beginning. Nature 431: 915-916, 2004.

8. Ponting CP and Belgard TG: Transcribed dark matter: meaning or myth? Hum Mol Genet 19: R162-R168, 2010.

9. Brosnan CA and Voinnet O: The long and the short of noncoding RNAs. Curr Opin Cell Biol 21: 416-425, 2009.

10. Mattick JS: Non-coding RNAs: the architects of eukaryotic complexity. EMBO Rep 2: 986-991, 2001. 
11. Costa FF: Non-coding RNAs: new players in eukaryotic biology. Gene 357: 83-94, 2005.

12. Lu J, Getz G, Miska EA, et al: MicroRNA expression profiles classify human cancers. Nature 435: 834-838, 2005.

13. Nelson KM and Weiss GJ: MicroRNAs and cancer: past, present and potential future. Mol Cancer Ther 7: 3655-3660, 2008.

14. Farazi TA, Spitzer JI, Morozov P and Tuschl T: miRNAs in human cancer. J Pathol 223: 102-115, 2011.

15. Huarte $M$ and Rinn JL: Large non-coding RNAs: missing links in cancer? Hum Mol Genet 19: R152-R161, 2010.

16. Khalil AM, Guttman M, Huarte M, et al: Many human large intergenic noncoding RNAs associate with chromatin-modifying complexes and affect gene expression. Proc Natl Acad Sci USA 106: 11667-11672, 2009.

17. Spitale RC, Tsai MC and Chang HY: RNA templating the epigenome: long noncoding RNAs as molecular scaffolds. Epigenetics 6: 539-543, 2011.

18. Rinn JL, Kertesz M, Wang JK, et al: Functional demarcation of active and silent chromatin domains in human HOX loci by noncoding RNAs. Cell 129: 1311-1323, 2007.

19. Woo CJ and Kingston RE: HOTAIR lifts noncoding RNAs to new levels. Cell 129: 1257-1259, 2007.

20. Gupta RA, Shah N, Wang KC, et al: Long non-coding RNA HOTAIR reprograms chromatin state to promote cancer metastasis. Nature 464: 1071-1076, 2010.

21. Chisholm KM, Wan Y, Li R, Montgomery KD, Chang HY and West RB: Detection of long non-coding RNA in archival tissue: correlation with polycomb protein expression in primary and metastatic breast carcinoma. PloS one 7: e47998, 2012.

22. Endo H, Shiroki T, Nakagawa T, et al: Enhanced expression of long non-coding RNA HOTAIR is associated with the development of gastric cancer. PloS One 8: e77070, 2013.

23. Yang Z, Zhou L, Wu LM, et al: Overexpression of long non-coding RNA HOTAIR predicts tumor recurrence in hepatocellular carcinoma patients following liver transplantation. Ann Surg Oncol 18: 1243-1250, 2011.

24. Milhem MM, Knutson T, Yang S, et al: Correlation of MTDH/AEG-1 and HOTAIR expression with metastasis and response to treatment in Sarcoma patients. J Cancer Sci Ther S5: pii004, 2011.

25. Ono $\mathrm{H}$, Motoi $\mathrm{N}$, Nagano $\mathrm{H}$, et al: Long noncoding RNA HOTAIR is relevant to cellular proliferation, invasiveness and clinical relapse in small-cell lung cancer. Cancer Med 3: 632-642, 2014

26. Li D, Feng J, Wu T, et al: Long intergenic noncoding RNA HOTAIR is overexpressed and regulates PTEN methylation in laryngeal squamous cell carcinoma. Am J Pathol 182: 64-70, 2013.

27. Kim K, Jutooru I, Chadalapaka G, et al: HOTAIR is a negative prognostic factor and exhibits pro-oncogenic activity in pancreatic cancer. Oncogene 32: 1616-1625, 2013.

28. Sparmann A and van Lohuizen M: Polycomb silencers control cell fate, development and cancer. Nature reviews. Cancer 6 : 846-856, 2006.

29. Orlando V: Polycomb, epigenomes and control of cell identity. Cell 112: 599-606, 2003

30. Mohammad HP, Cai Y, McGarvey KM, et al: Polycomb CBX7 promotes initiation of heritable repression of genes frequently silenced with cancer-specific DNA hypermethylation. Cancer Res 69: 6322-6330, 2009.

31. Kleer CG, Cao Q, Varambally S, et al: EZH2 is a marker of aggressive breast cancer and promotes neoplastic transformation of breast epithelial cells. Proc Natl Acad Sci USA 100 11606-11611, 2003

32. Tsai MC, Manor O, Wan Y, et al: Long noncoding RNA as modular scaffold of histone modification complexes Science 329: 689-693, 2010

33. Lu L, Zhu G, Zhang C, et al: Association of large noncoding RNA HOTAIR expression and its downstream intergenic $\mathrm{CpG}$ island methylation with survival in breast cancer. Breast Cancer Res Treat 136: 875-883, 2012.

34. Sorensen KP, Thomassen M, Tan Q, et al: Long non-coding RNA HOTAIR is an independent prognostic marker of metastasis in estrogen receptor-positive primary breast cancer. Breast Cancer Res Treat 142: 529-536, 2013.

35. Lv XB, Lian GY, Wang HR, Song E, Yao H and Wang MH: Long noncoding RNA HOTAIR is a prognostic marker for esophageal squamous cell carcinoma progression and survival. PloS One 8: e63516, 2013.
36. Ge XS, Ma HJ, Zheng XH, et al: HOTAIR, a prognostic factor in esophageal squamous cell carcinoma, inhibits WIF-1 expression and activates Wnt pathway. Cancer Sci 104: 1675-1682, 2013.

37. Li X, Wu Z, Mei Q, Guo M, Fu X and Han W: Long non-coding RNA HOTAIR, a driver of malignancy, predicts negative prognosis and exhibits oncogenic activity in oesophageal squamous cell carcinoma. Br J Cancer 109: 2266-2278, 2013

38. Liu XH, Liu ZL, Sun M, Liu J, Wang ZX and De W: The long non-coding RNA HOTAIR indicates a poor prognosis and promotes metastasis in non-small cell lung cancer. BMC Cancer 13: 464, 2013.

39. Egeblad M, Rasch MG and Weaver VM: Dynamic interplay between the collagen scaffold and tumor evolution. Curr Opin Cell Biol 22: 697-706, 2010.

40. Nakagawa T, Endo H, Yokoyama M, et al: Large noncoding RNA HOTAIR enhances aggressive biological behavior and is associated with short disease-free survival in human non-small cell lung cancer. Biochem Biophys Res Commun 436: 319-324, 2013.

41. Xu ZY, Yu QM, Du YA, et al: Knockdown of long non-coding RNA HOTAIR suppresses tumor invasion and reverses epithelial-mesenchymal transition in gastric cancer. Int J Biol Sci 9: 587-597, 2013

42. Ishibashi M, Kogo R, Shibata K, et al: Clinical significance of the expression of long non-coding RNA HOTAIR in primary hepatocellular carcinoma. Oncol Rep 29: 946-950, 2013.

43. He X, Bao W, Li X, et al: The long non-coding RNA HOTAIR is upregulated in endometrial carcinoma and correlates with poor prognosis. Int J Mol Med 33: 325-332, 2014.

44. Chiyomaru T, Yamamura S, Fukuhara S, et al: Genistein inhibits prostate cancer cell growth by targeting miR-34a and oncogenic HOTAIR. PloS One 8: e70372, 2013.

45. Nie Y, Liu X, Qu S, Song E, Zou H and Gong C: Long non-coding RNA HOTAIR is an independent prognostic marker for nasopharyngeal carcinoma progression and survival. Cancer Sci 104: 458-464, 2013

46. Stratford JK, Bentrem DJ, Anderson JM, et al: A six-gene signature predicts survival of patients with localized pancreatic ductal adenocarcinoma. PLoS Med 7: e1000307, 2010.

47. Kogo R, Shimamura T, Mimori K, et al: Long noncoding RNA HOTAIR regulates polycomb-dependent chromatin modification and is associated with poor prognosis in colorectal cancers. Cancer Res 71: 6320-6326, 2011.

48. Tang L, Zhang W, Su B and Yu B: Long noncoding RNA HOTAIR is associated with motility, invasion and metastatic potential of metastatic melanoma. Biomed Res Int 2013: 251098, 2013.

49. Zhang JX, Han L, Bao ZS, et al: HOTAIR, a cell cycle-associated long noncoding RNA and a strong predictor of survival, is preferentially expressed in classical and mesenchymal glioma. Neuro Oncol 15: 1595-1603, 2013.

50. Li Z, Li C, Liu C, Yu S and Zhang Y: Expression of the long non-coding RNAs MEG3, HOTAIR and MALAT-1 in non-functioning pituitary adenomas and their relationship to tumor behavior. Pituitary 18: 42-47, 2015.

51. Kalluri R and Weinberg RA: The basics of epithelial-mesenchymal transition. J Clin Invest 119: 1420-1428, 2009.

52. Chen K, Huang YH and Chen JL: Understanding and targeting cancer stem cells: therapeutic implications and challenges. Acta Pharmacol Sin 34: 732-740, 2013.

53. Padua Alves C, Fonseca AS, Muys BR, et al: Brief report: The lincRNA HOTAIR is required for epithelial-to-mesenchymal transition and stemness maintenance of cancer cell lines. Stem Cells 31: 2827-2832, 2013.

54. Rangel MC, Karasawa H, Castro NP, Nagaoka T, Salomon DS and Bianco C: Role of Cripto-1 during epithelial-to-mesenchymal transition in development and cancer. Am J Pathol 180: 2188-2200, 2012

55. Lee S, Hong S, Kim S and Kang S: Ataxin-1 occupies the promoter region of E-cadherin in vivo and activates CtBP2-repressed promoter. Biochim Biophys Acta 1813: 713-722, 2011.

56. Jeanes A, Gottardi CJ and Yap AS: Cadherins and cancer: how does cadherin dysfunction promote tumor progression? Oncogene 27: 6920-6929, 2008.

57. Stemmler MP: Cadherins in development and cancer. Mol Biosyst 4: 835-850, 2008

58. Nakajima M, Fukuchi M, Miyazaki T, Masuda N, Kato H and Kuwano H: Reduced expression of Axin correlates with tumour progression of oesophageal squamous cell carcinoma. $\mathrm{Br}$ J Cancer 88: 1734-1739, 2003. 
59. Kouzarides T: Chromatin modifications and their function. Cell 128: 693-705, 2007.

60. Shi Y, Lan F, Matson C, et al: Histone demethylation mediated by the nuclear amine oxidase homolog LSD1. Cell 119: 941-953, 2004.

61. Pickart CM: Mechanisms underlying ubiquitination. Annu Rev Biochem 70: 503-533, 2001.

62. Yoon JH, Abdelmohsen K, Kim J, et al: Scaffold function of long non-coding RNA HOTAIR in protein ubiquitination. Nat Commun 4: 2939, 2013.
63. Hebner C, Weaver VM and Debnath J: Modeling morphogenesis and oncogenesis in three-dimensional breast epithelial cultures. Annu Rev Pathol 3: 313-339, 2008.

64. Bhan A, Hussain I, Ansari KI, Bobzean SA, Perrotti LI and Mandal SS: Bisphenol-A and diethylstilbestrol exposure induces the expression of breast cancer associated long noncoding RNA HOTAIR in vitro and in vivo. J Steroid Biochem Mol Biol 141: 160-170, 2014. 